Fecha de recepción: junio 2018

Fecha de aceptación: marzo 2020

Versión final: septiembre 2020

\section{Organicismo: morfología y materialidad como expresión comunicante en un espacio arquitectónico}

Ricardo José Viveros Baez *

Resumen: A partir de la arquitectura orgánica, entendida como una filosofía que promueve la armonía entre el hábitat humano y el entorno natural, se marca un quiebre sustancial con las composiciones formales tradicionales, y desde donde se aprovechó las propiedades de nuevos materiales y nuevas técnicas productivas. Este movimiento surge en oposición al racionalismo o funcionalismo, manifestándose en las primeras décadas del siglo XX y con ello se compromete el diseño de objetos y particularmente el diseño de mobiliario de interiores, basado en estructuras orgánicas, y de una forma de diseño que abre fronteras entre lo material y lo sentimental, cuya fuente de vivencias es la naturaleza.

La gran diversidad de soluciones -presentes en nuestro entorno natural- a problemas estructurales, en términos de energía y funcionalidad, ya han sido probados durante millones de años, y en ella podemos encontrar todo tipo de patrones y combinaciones de formas, colores y texturas que son el fundamento del diseño orgánico como una fuente inagotable de inspiración para diseñadores. Se constituye como una poderosa herramienta y fuente de inspiración sin fin, unida por los principios de la vida misma, tratando de imitar las formas, texturas o funciones de los organismos vivos para alcanzar soluciones de diseño óptimas.

En el presente trabajo se muestra un breve recorrido por los inicios del diseño orgánico, particularmente el diseño de mobiliario, recorriendo algunos clásicos representativos del diseño nórdico y estadounidense, desde la relación forma-materia.

Luego se reconoce uno de los principales exponentes del diseño orgánico: Alvar Aalto, arquitecto y diseñador finlandés, a través de dos de sus creaciones más icónicas; el proyecto arquitectónico Sanatorio de Paimio y la silla 41. La idea central del Sanatorio, era un edificio que favoreciera la curación y rehabilitación de enfermos de tuberculosis. Este proyecto fue concebido integralmente, lo que le llevó a la definición de un mobiliario específico. Producto de ello es la silla número 41, más tarde conocida como silla Paimio de la cual se da cuenta de sus características morfológicas y de materialidad. Finalmente se establece una relación donde el objeto se conecta visual y funcionalmente con el contexto de su emplazamiento interior del conjunto arquitectónico.

Palabras clave: Organicismo - diseño orgánico - Sanatorio Paimio - silla 41 - Aalvar Alto.

[Resúmenes en inglés y portugués en las páginas 95-96]

${ }^{(*)}$ Diseñador con Mención en Equipos y Sistemas Universidad de Chile, Sede Valparaíso, Chile. Magíster en Pedagogía Universitaria, Universidad Andrés Bello, Valparaíso, Chile. 
Diplomado en Docencia Universitaria en Ingeniería, Universidad Técnica Federico Santa Maria, Valparaíso, Chile. Candidato a Doctor en Programa de Doctorado en Diseño en la Universidad de Palermo, Buenos Aires, Argentina. Profesor, Programa de Inserción Temprana, PIT. Carrera de Ingeniería en Diseño de Productos, UTFSM. Director, Escuela de Diseño de la Universidad Marítima de Chile, Viña del Mar - 1999 - 2007. Integra equipo asesor del Centro de Innovación más Productos, CIP, dependiente de la carrera de Ingeniería en Diseño de Productos de la UTFSM. Miembro del Comité de Arbritaje en Diseño y Miembro de la Comisión Latinoamericana de Postgrado. Centro de Estudios en Diseño y Comunicación. Universidad de Palermo, Argentina. Evaluador para Quacquarelli Symonds Limited, QS. Consultor Proyecto de Marketing Territorial para el Gobierno Regional de la Regio de los Ríos.

\section{Introducción}

Entendiendo que la disciplina del diseño responde a una actividad proyectual de carácter contextual y cultural, circunscrita a la creatividad que busca, mediante un proceso deliberado, un resultado formal para dar respuestas a determinadas necesidades del hombre en sociedad, ésta ha contribuido a la configuración del medio social ambiental en el que se desenvuelve la vida cotidiana de las personas, que no es única y homogénea, sino todo lo contrario, heterogénea, diversa y plural.

La disciplina como una actividad creadora asume como objetivo el determinar las cualidades formales de los objetos -haciendo referencia particularmente al diseño industrial-que serán materializados a través de tecnologías productivas. Estas cualidades no están referidas en sólo a aspectos externos, sino principalmente a aquellas relaciones estructurales y funcionales que convierten un sistema en una actividad coherente tanto desde el punto de vista del fabricante como del usuario. Por lo tanto, el proyectar la forma no se reduce solo a determinar la apariencia externa de los objetos sino que supone integrar y articular todos los factores que, de una manera $u$ otra, participan en el proceso de materialización del producto, y responde, desde una mirada vitruviana, a la triada utilitas - firmitas - venusta, como actores funcionales, técnicos y simbólicos.

Cuando se habla del diseño, se habla de un pensamiento relacional y semiótico, y en donde el diseñador se presenta como un elaborador de objetos con los que el hombre interactúa de forma sensible y emocional. A partir de ello es que, en el transcurso de la historia, han surgido diversas tendencias orientadas a "humanizar" el diseño, pero ¿qué fuente de inspiración podría conducir a una nueva concepción de diseños de productos totalmente frescos e innovadores que mejoren la calidad de vida, objetos que sean simples pero sofisticados, elegantes, inteligentes y respetuosos del medio ambiente?

Tal es el caso del Organicismo que surge de la arquitectura orgánica como una filosofía que promueve la armonía entre el hábitat humano y el entorno natural.

Este movimiento surge en oposición al racionalismo o funcionalismo; aparece en las primeras décadas del siglo XX, marcando un quiebre sustancial con las composiciones formales tradicionales, y desde donde se aprovechó las propiedades de los nuevos materiales 
industriales, especialmente el hormigón armado, el acero laminado y el vidrio plano en grandes dimensiones. Se caracterizó por la asimetría de sus plantas y secciones ortogonales, las fachadas carentes de elementos decorativos y la horizontalidad de grandes ventanales divididos por perfiles de acero. Los interiores tienden, por lo general, a ser luminosos y diáfanos. Los mayores exponentes de este movimiento se ubican a partir de la década de 1920, en los propuestas de arquitectos como Walter Gropius, Mies van der Rohe, Le Corbusier.

El organicismo, según afirman Araya, Alfaro y Andonegui (2007):

Se basa en la metáfora raíz de los procesos orgánicos inherentes a todos los sistemas vivos y en evolución. Se concibe como un proceso dialéctico en el que se confrontan los fenómenos mediante oposiciones y contradicciones que dan lugar a cambios cualitativos en vías de una mayor complejidad e integración. La causa final es una forma teleológica de razonamiento causal, a través de la cual se entiende que los fenómenos están en un proceso continuo de cambio evolutivo y estructural hacia un todo mayor, aunque inespecificable (p. 86).

De esta corriente organicista relacionadas con la naturaleza, se desprende el Diseño Orgánico como un movimiento impulsador de nuevas concepciones, basado en estructuras orgánicas, y de una forma de diseño que abre fronteras entre lo material y lo sentimental, que tiene como fuente de vivencias a la naturaleza. La generosa diversidad de nuestro entorno natural contiene un sinfín de soluciones a problemas estructurales, en términos de energía y funcionalidad, todos sometidos a pruebas y evolucionados durante millones de años. Parece lógico pensar que sería más fácil aprovechar el vasto archivo de información que la naturaleza nos ofrece que explotar todos sus recursos incontrolablemente. La belleza de la naturaleza está presente en el consciente y el inconsciente colectivo de toda la humanidad, y ha estado presente desde el comienzo de los tiempos. En la naturaleza podemos encontrar todo tipo de patrones y combinaciones de formas, colores y texturas. Estos fascinantes modelos son el fundamento del diseño orgánico como una fuente inagotable de inspiración para diseñadores y artistas. En el diseño de productos, es una poderosa herramienta y fuente de inspiración sin fin, unida por los principios de la vida misma, tratando de imitar las formas, texturas o funciones de los organismos vivos para alcanzar soluciones de diseño óptimas. Hoy, el diseño orgánico, en una concepción biomimética, está empezando a recibir reconocimiento mundial. Cada vez más ingenieros, diseñadores $y$ arquitectos se inspiran en los procesos naturales para desarrollar diseños que mejoran la calidad de vida del ser humano y el planeta.

\section{Morfología y materialidad}

En ese enfoque orgánico del diseño es crucial la manera en que los elementos individuales, como objetos o muebles, se vinculan visual y funcionalmente con el contexto de su emplazamiento interior y el conjunto de su entorno. En esta condición responde a un principio hilemórfico ${ }^{2}$ en tanto se halla constituido por dos dimensiones esenciales; Materia 
y Forma. La materia como el sustrato básico de toda realidad, carente de forma, pero sin embargo no puede darse sin la forma, así como la forma no puede darse sin la materia. El uno no puede estar sin el otro. Configuran una unidad, inseparables e indisolubles (Ruiz 2015, p. 71).

En esta dialéctica materia y forma, el organicismo, tanto en Europa como en Estados Unidos, convive durante la década cincuenta con la tendencia racional-funcionalista. A principios de los cuarenta, el arquitecto y diseñador industrial Eliot Noyes ${ }^{3}$ organizó el concurso Organic Design in Home Furnishing en el Museum of Modern Art de Nueva York. En él destacó -en la categoría "asientos para un salón"- la propuesta presentada por los arquitectos Eero Saarinen -finlandés- y Charles Eames -estadounidense-, quienes presentaron la "silla orgánica" (Ver Figura 1), un diseño revolucionario tanto por su forma, por el material -madera contrachapada y madera prensada-, como por la forma de producción; moldeo de forma única que inauguraba el concepto de contacto y soporte continuos. Sin embargo, esta propuesta, muy avanzada para su tiempo, vio limitada su opción de fabricación seriada. Ello fue posible después de 1950, cuando el desarrollo tecnológico fue suficiente como para fabricarla en grandes cantidades (no era posible hacerlo, dado los elevados costos de producción y a la guerra). Está pensada como pequeño y confortable sillón de lectura, tiene como principal característica que el asiento, respaldo y brazos forman una sola pieza en forma de caparazón. Se compone de una carcasa laminada, acolchado en espuma de poliuretano y tapizado en tela. La base está hecha en madera de fresno negro y roble natural. Hoy en día está disponible en versión con y sin reposacabezas.

Esta tendencia influyó rápidamente en el diseño de mobiliario, resultando ser cómodos, económicos y ergonómicos, lo que permitió un fuerte auge y desarrollo en Estados Unidos destacándose los aportes de los arquitectos y diseñadores Charles y Ray Eames, Henry Bertoia o Eero Saarinen. Este último destacó con su famosa "silla tulipán" (Ver Figura 2), creada en 1956, y que va más allá de una sola pieza de mobiliario, sino que dio cabida a una línea compuesta de sillas con apoya-brazos, silla sin apoya-brazos, banquetas y mesas de diversos tamaños. Todos los componentes de esta línea se destacan por dejar de lado la idea de patas y en vez de eso poseen un solo pedestal o una pata única. De esta manera, se aprovecha al máximo el espacio residual por debajo de las sillas y mesas, y dejan este espacio libre para el usuario. Esta fue fabricada en metal fundido y asiento acolchado, logrando minimizar la profusión de patas de sillas en los espacios interiores. La unidad de estilo de toda la línea se ve reflejada en sus materiales y formas. La personalización de cada pieza va de la mano del tapizado de sus cojines, donde existe la posibilidad de utilizar diferentes telas y colores.

Por otra parte, en Europa, Arne Jacobsen, arquitecto y diseñador industrial, fue uno de los primeros que destacó en las propuestas de mobiliario de carácter orgánico, entre sus aportes se destacan sus sillas de la Serie 7 (Ver Figura 3) diseñada en 1955, inicialmente para la exposición de diseño nórdico realizada en Helsingborg, Suecia, en el mismo año. Fue creada en chapa moldeada a presión, apilable de cuatro patas, es la culminación de la utilización de la técnica de laminación desarrollada en los años 20 por Soren Hansen 4 Jacobsen explotó las posibilidades de laminación a la perfección lo que resulta en la forma icónica de esta silla ligera y apilable. 


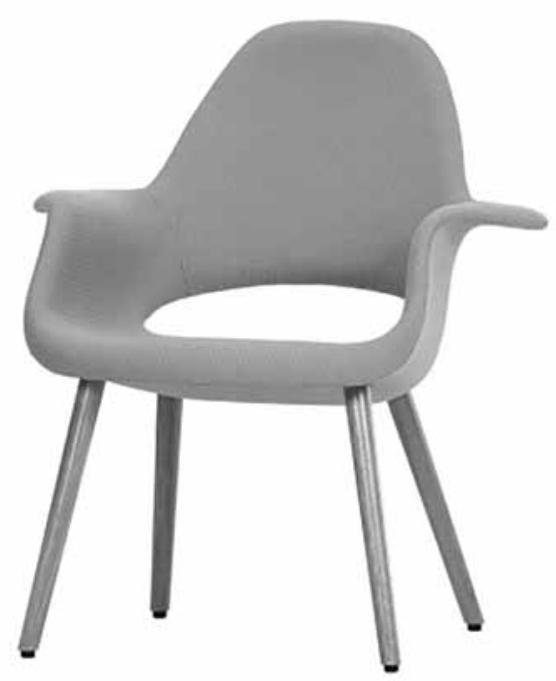

Figura 1.

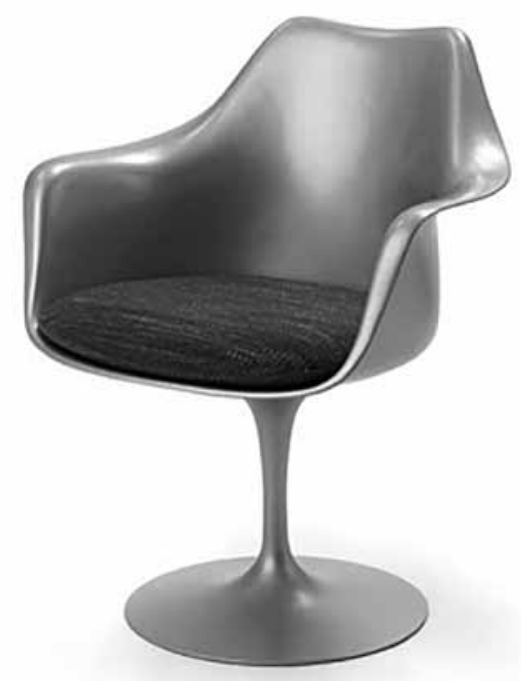

Figura 2.

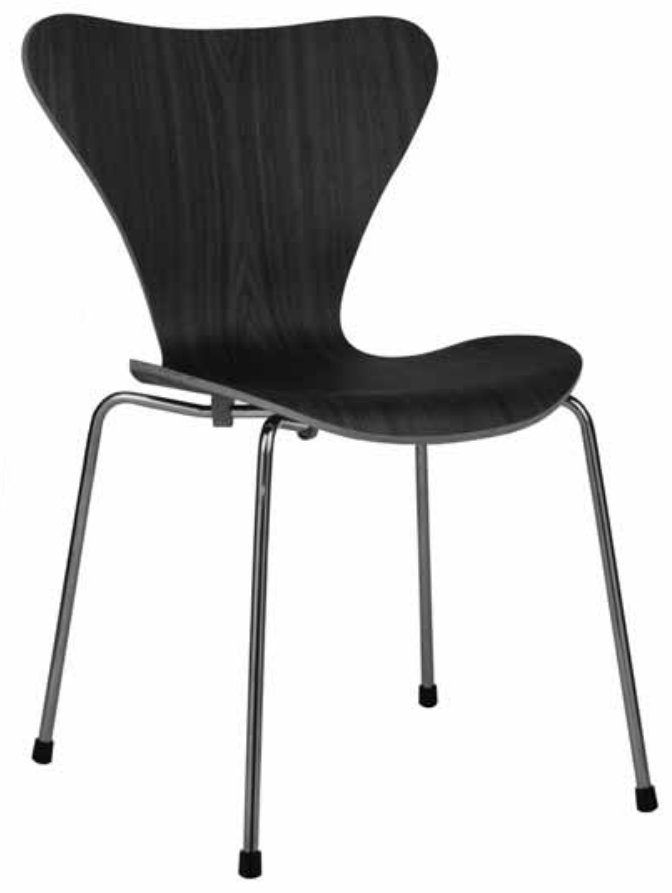

Figura 3.

Figura 1. La silla orgánica de los arquitectos Charles Eames y Eero Saarinen creada en 1940 para el concurso de Diseño Orgánico del Mobiliario para el Hogar (Organic Design in Home Furnishings) en el Museo de Arte Moderno de Nueva York (MoMA), organizado para contrarrestar el estancamiento en el diseño de mobiliario de la época. Fuente: http://decofilia. com/blog/1940-silla-organicaorganic-chair/

Figura 2. La Tulip Chair, diseñada en 1956 por Eero Saarinen. Fuente: https://www.disenoyarquitectura. net/2008/12/un-icono-de-laepoca-pop-la-silla-tulip.html Figura 3. Silla serie 7 diseñada en 1955 por Jacobsen. Fuente: http://historia-disenio-industrial. blogspot.cl/2014/08/serie-7.html 
Se destacan igual sus sillas de estructuras metálicas recubiertas de acolchados, como la silla Swan (Ver Figura 4) creada en 1958, formó parte del mobiliario del Hotel SAS de Copenhagen, caracterizándose por la ausencia de línea rectas, lo que la hace muy orgánica -su forma se asocia a un tulipán-y original, estaba realizada en lana de Cashmerede color rojo, y su base era de aluminio cromado. En su concepción tuvo un alto grado de innovación, cuando la silla moderna ya había sido inventada años atrás por Le Corbusier, Mies van der Rohe y Marcel Breuer, con sus curvas sinuosas representa todo lo que es positivo en diseño: estética, humanidad y confort, siempre necesarios en una ambiente de interior. En sus rasgos morfológicos se simboliza la estética danesa, minimalismo y elegancia en la forma. Así también la silla Egg (Ver Figura 5), de grandes dimensiones y que lleva la forma del material al límite, está fabricada con un armazón de madera contrachapada de abedul recubierta de almohadillas de espuma y tela, una técnica de la que Jacobsen fue pionero. En su concepción combinó la forma sensual y estética con elementos de comodidad y funcionalidad. Fue fabricada expresamente para el vestíbulo del hotel Royal de Copenhague en 1958, el diseño de esta silla ha representado la estética del modernismo, contribuyendo significantemente en el diseño nórdico. Con el objetivo de ofrecer privacidad, esta silla tiene un aspecto acogedor que se integra en cualquier entorno convirtiéndose en el punto central de cualquier espacio.

Junto a ello destacan, además, las lámparas para Louis Poulsen 5 . La AJ (Ver Figura 6), fue diseñada en 1957 para una de las obras maestras de Jacobsen, el Radisson SAS Royal Hotel de Copenhague, se caracteriza por su diseño orgánico, basado en el haz de luz que proyecta la bombilla que se haya en el interior del cuerpo de la lámpara de cobre esmaltado, sintetizando la fuerte estética rectilínea del edificio con el vocabulario más orgánico de las famosas sillas Swan y Egg, que también fueron diseñadas para el proyecto. Supone la evolución en el diseño sobre todo de las lámparas de despacho, dando paso a nuevas formas orgánicas, aunque sin obviar la estructura básica de este tipo de lámparas. Sus rasgos dan cuenta de una base de cinc horadada con forma de huevo -el agujero se debe a que originalmente estaba pensada para alojar un cenicero- sale un delgado pie de acero inclinado y una pantalla de acero centrifugado que emite la luz hacia abajo y puede moverse para ajustar la dirección del haz. La pantalla está pintada en blanco por la parte interior para garantizar la emisión de una luz suave y confortable.

Gran éxito por sus propuestas innovadoras, tuvo el arquitecto y diseñador industrial danés Verner Panton, particularmente su aportaciones presentadas en la Feria del Mueble de Colonia de 1960, para posteriormente conseguir fabricar una de las sillas más destacadas del siglo XX, una silla en voladizo; la silla Panton (Ver Figura 7), la primera realizada de una sola pieza en plástico inyectado a doble cara, y que muestra la evolución de los materiales aplicados al diseño.

Es evidente el parecido con la Silla Zig-Zag de Rietveld, sin embargo se trata de un mueble totalmente diferente gracias al plástico modelable, creando un diseño más limpio y orgánico que la predecesora realizada en madera y caracterizada por sus formas inanimadas. Y es que la Panton, además de suntuosa y sexy, por sus esculpidas curvas, es también un símbolo del optimismo de la década de los '60. 


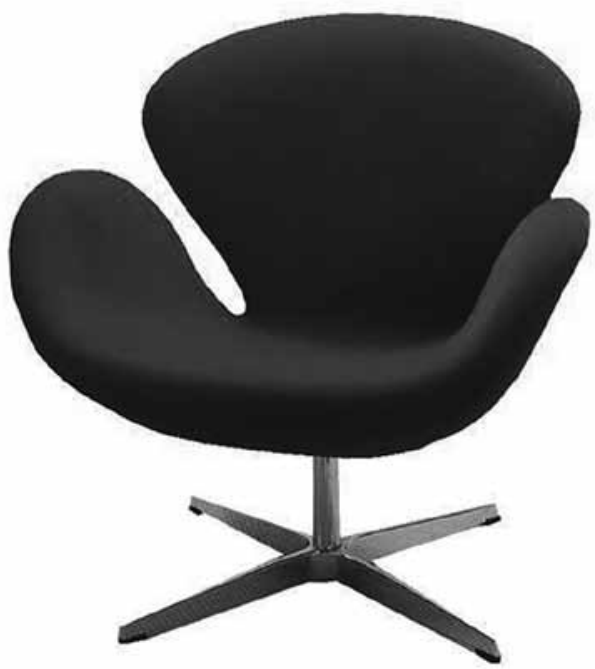

Figura 4.

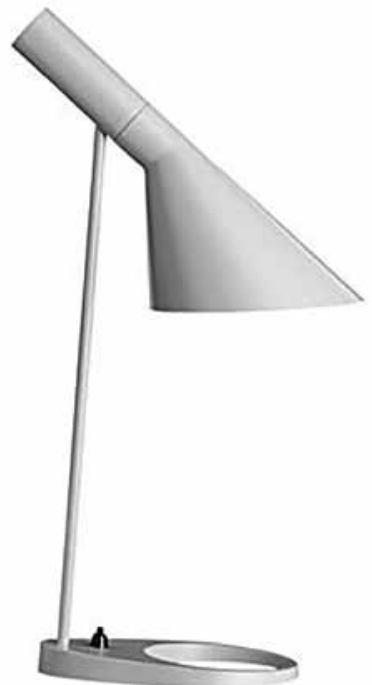

Figura 6.

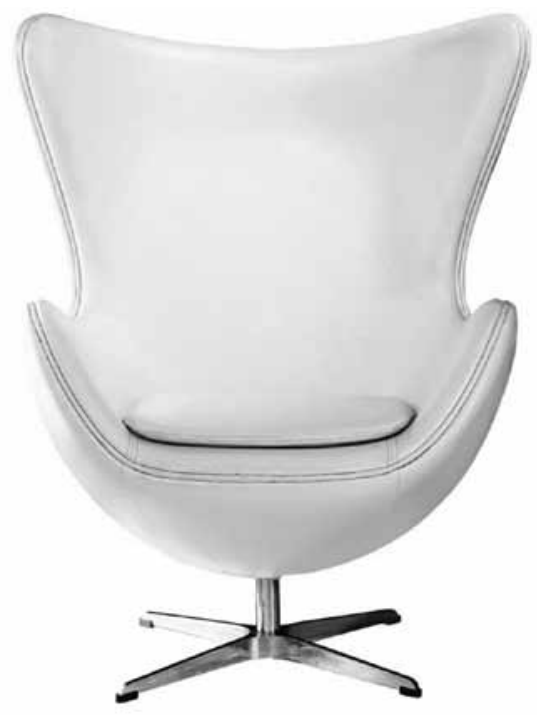

Figura 5.

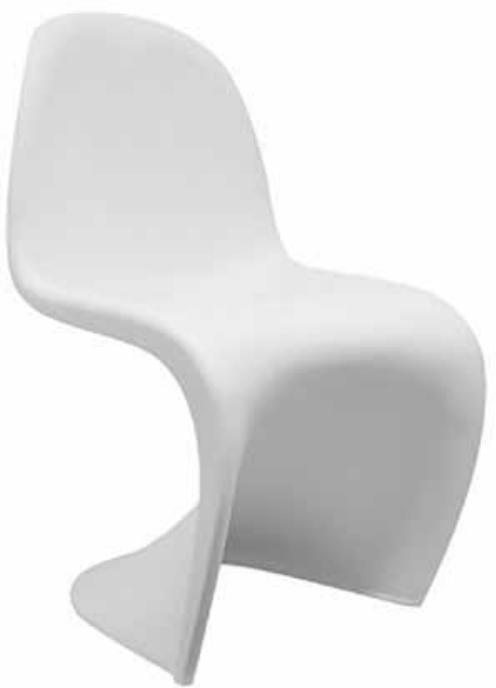

Figura 7.

Figura 4. Silla Swan diseñada en 1958 por Jacobsen. Fuente: https:/www.disenoyarquitectura.net/2009/07/sillaswan-de-jacobsen.html. Figura 5. Silla Egg diseñada en 1958 por Jacobsen. Fuente: https://www.disenoyarquitectura. net/2009/08/sillon-egg-de-arne-jacobsen.html. Figura 6. Lámpara AJ diseñada en 1957 por Jacobsen. Fuente: https:// www.disenoyarquitectura.net/2009/06/lampara-aj-de-arne-jacobsen.html. Figura 7. Silla Panton diseñada en 1960 por Verner Panton. Fuente: https://www.disenoyarquitectura.net/2009/06/silla-panton-un-diseno-para-el-dia-dia.html 
La silla Panton es una de las más conocidas del Movimiento Moderno, inspirada en la visión de una pila de cubos de plástico, el objetivo era conseguir una silla cómoda, elegante, que pudiera utilizarse en cualquier espacio y que, además, fuese económica. Como resultado se obtuvo una silla convertida en un icono de la modernidad: mimética y camaleónica, que encaja en decoraciones de casi cualquier estilo.

Dado su versatilidad, se puede encontrar en oficinas, estudios de televisión, restaurantes y un largo etcétera, lo que la hace una silla polivalente. Esa polivalencia se traslada a su uso interior o exterior, ya que al ser de una sola pieza, es práctica tanto para el interior como para los espacios abiertos.

\section{El Sanatorio de Paimio y la silla 41 de Aalvar Alto}

Siendo el estilo orgánico un movimiento que surge de la arquitectura, derivado del funcionalismo o racionalismo, es promovido fundamentalmente por arquitectos escandinavos y por el arquitecto norteamericano Frank Lloyd. No obstante se destaca la figura del diseñador de muebles estadounidense Gustav Stickley, de los arquitectos Antoni Gaudí -español-, Louis Sullivan y Bruce Goff -estadounidenses-, Bruno Zevi -italiano-, Friedrich Hundertwasser -austríaco-, Samuel Flores Flores -uruguayo-, Alvar Aalto -finlandés-, Imre Makovecz -húngaro-, Javier Senosiain - mexicano- y el belga Antón Alberts. Siendo los mayores exponentes de la denominada arquitectura orgánica, establecen el principio estructural de la forma a través del diseño comprendiendo e integrando al sitio, los edificios, los mobiliarios, y los alrededores para que se conviertan en parte de una composición unificada y correlacionada, en tanto se establece una coherencia armónica entre las partes y el todo. En esta integración de elementos compositivos se advierte una primera lectura de una organización formal que busca incrementar la coherencia interna, debido a que la convergencia simultánea es conducente al reforzamiento de las características de los elementos considerados.

Este movimiento adopta varias de las propuestas del racionalismo, como son la planta libre, el predominio de lo útil sobre lo meramente ornamental, la consideración de los adelantos tecnológicos - particularmente los materiales- de la era industrial a la arquitectura con el propósito de aportar nuevos valores.

Uno de los principales propugnadores de esta corriente es Hugo Alvar Henrik Aalto (18981976); arquitecto y diseñador finlandés, importante figura del Movimiento Moderno. Sus ideas fundamentales están orientadas a una mayor preocupación por la vida del hombre, poniendo más atención a éste que a la arquitectura, en donde la atención del arquitecto no debe limitarse a las estructuras y a la disposición de los ambientes, sino que debe extenderse a los problemas psicológicos y vitales del hombre.

Se concibe un nuevo entendimiento de los espacios de interiores, ya que la arquitectura racionalista propugna una adaptación de los volúmenes a las necesidades de planta, esos volúmenes están dominados por un estilo cubista, de formas tetraédricas, en las que se van encajando las necesidades, buscando además con ello una simplificación estructural y constructiva. Aalto comienza por el estudio de los ambientes, de los recorridos, de los movimientos del usuario llegando a la creación de espacios que considera más indicados, 
y a partir de ello le incorpora una envolvente adecuada. Las soluciones técnicas y estructurales se ajustan a esos espacios, dando lugar normalmente a formas más complicadas, no repetidas, económicamente más costosas y difíciles de industrializar.

Se advierte una concepción morfológica exploratoria entre la configuración espacial y el valor funcional-social estableciendo una condición adaptativa a la condición formal.

En este aspecto, el organicismo abandona uno de los postulados del racionalismo, creando en cambio, cuando es bien interpretado, realizaciones más humanas, de modo que son más personales las obras arquitectónicas orgánicas, son difíciles de imitar y por lo tanto es más propio hablar de un planteamiento que de un estilo de arquitectura orgánica.

Como obras maestras de Aalto se encuentra la biblioteca de Viipuri (1930), el pabellón finlandés en la Exposición de Nueva York de 1939y el icónico Sanatorio para tuberculosos de Paimio, Finlandia, cuyo periodo de construcción fue entre 1929 y 1933 (Ver Figura 8). Este proyecto es el resultado de un concurso ganado en 1929, colocando a Finlandia en el mapa de la arquitectura moderna. Finlandia quedó, tras la guerra civil de independencia de Rusia en 1919, con una gran epidemia de tuberculosis y el tratamiento conocido en aquella época era una rutina que incluía baños de sol y largas horas de descanso respirando aire fresco.

De acuerdo con estos ideales higienistas, e inspirado por el Zonnestraal de Duiker ${ }^{6}$, Alvar Aalto proyectó este conjunto que se ubica en lo más alto de un idílico y aislado entorno al sureste de Finlandia, a unos $29 \mathrm{Kms}$ de la ciudad de Turku, el complejo de edificios que lo componen se abre y articula hacia el exterior, permitiendo vistas, ventilación y asoleamiento natural en todas las habitaciones y demás estancias (Ver Figuras 9 y 10). El conjunto, claramente funcional, tiene una aproximación humana muy propia de la arquitectura de Aalto que va más allá del funcionalismo técnico y que se percibe sobre todo en el detalle de sus elementos. Esta obra supone un claro punto de inflexión en la obra de Aalto, pasando de una producción caracterizada por el respeto a la tradición hacia una arquitectura plenamente relacionada ya con la cultura del Movimiento Moderno.

La idea principal para el Sanatorio, proyectado inicialmente para 296 pacientes, era un edificio que favoreciera la curación y rehabilitación de enfermos de tuberculosis, o en palabras del mismo Aalto, un edificio concebido como si de un "instrumento médico" se tratara. El conjunto está formado por zonas claramente diferenciadas, la de los pacientes y galerías o salas de reposo, la de las salas comunes y la de servicios, articulando los bloques que contienen dichas funciones según la orientación y vistas alrededor de un núcleo central de circulaciones. Las casas de médicos y empleados son tratadas como pabellones aislados, para dar privacidad y descanso a los trabajadores.

Aalto pone especial atención en la distribución, funcionamiento y orientación del bloque principal, contenedor de las habitaciones y salas de reposo para los enfermos, estas últimas situadas en el extremo de cada planta. Orientadas las primeras a sur-sureste y las salasterraza de reposo a pleno sur, Aalto consigue un máximo aprovechamiento de la luz y propiedades curativas del sol para facilitar la rehabilitación de los enfermos (Ver Figura 11). Esta característica morfológica con el manejo de las proporciones y de la iluminación tiende a estimular las condiciones de desplazamiento en tanto su temporalidad, así como los estados de anímicos en tanto su estabilidad o variabilidad. 


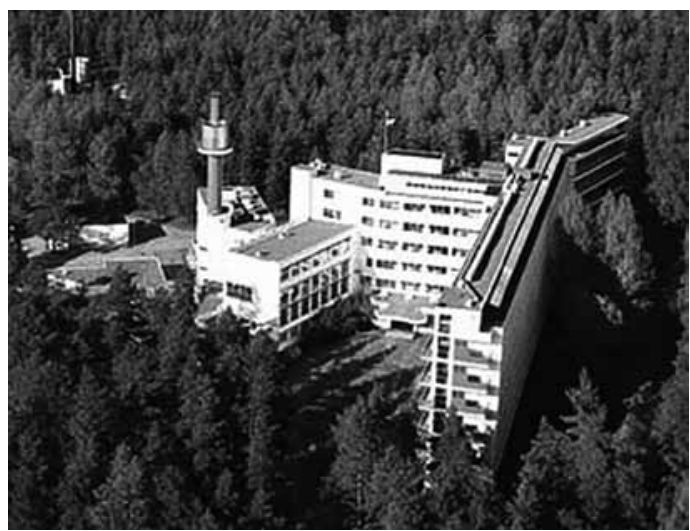

Figura 8.

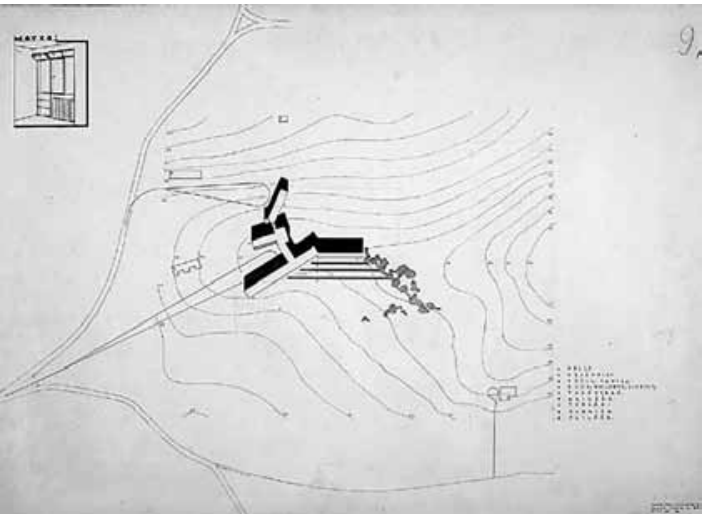

Figura 9.

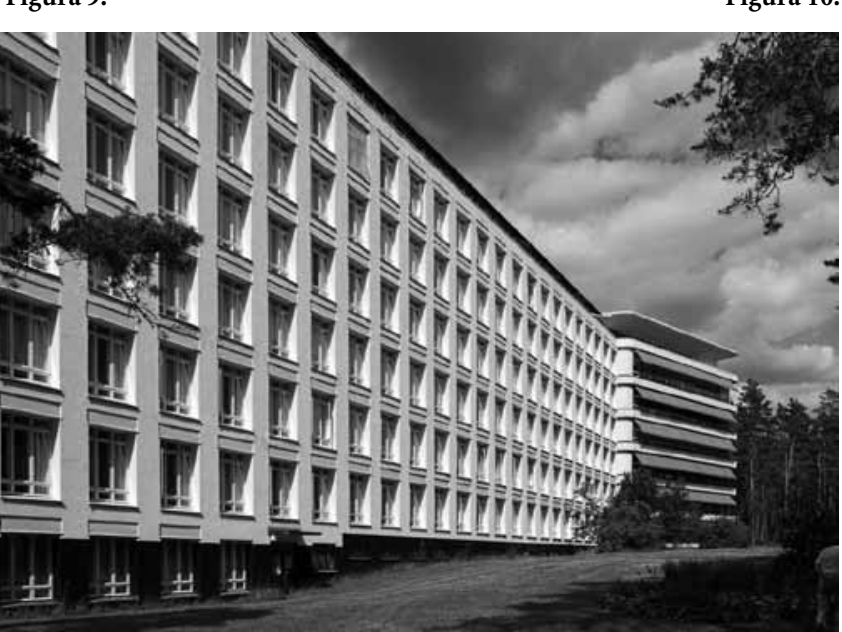

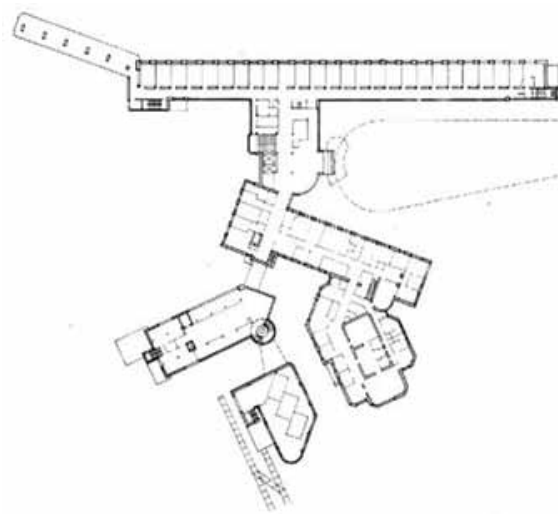

Figura 10.

Figura 8. Vista general del Sanatorio de Paimio, Finlandia. Fuente: https://es.wikiarquitectura. com/edificio/sanatorioantituberculoso-paimio/ Figura 9 y 10. Planos del conjunto de edificios del Sanatorio de Paimio. Fuente:https:// es.wikiarquitectura.com/edificio/ sanatorio-antituberculoso-paimio/ Figura 11. Orientación sur del Sanatorio de Paimio. Fuente: https://es.wikiarquitectura.com/ edificio/sanatorio-antituberculosopaimio/

Figura 11. 
También contempla una sala de reposo en cubierta, para 120 tumbonas (Ver Figura 12), que ocupa todo el largo de la superficie y corona el edificio, ofreciendo unas vistas inmejorables sobre el paisaje y cuyos jardines evitan el exceso de calor durante el verano. En el interior de las habitaciones, habilitadas para dos ocupantes, el detallado diseño de sus elementos proporciona una máxima comodidad al paciente, como por ejemplo a través de la disposición indirecta de luz artificial, el color del techo pintado en verde oscuro para evitar deslumbramientos, o también la colocación de la calefacción en el techo para evitar la radiación directa, y el cuidado diseño de los lavabos, cuya geometría minimiza el posible ruido provocado por su uso (Ver Figura 13).

Las estancias comunes más espaciosas, como son el comedor y el taller, están orientadas a sur y están provistas de toldos para un control solar. También estos espacios ofrecen diferentes orientaciones y visuales para provocar en el paciente una variedad de estímulos psíquicos.

La estructura del Sanatorio, compuesta de columnas de hormigón armado, está perfectamente modulada con los espacios interiores que alberga. Incluye otras paredes de carga, de 8 a 10 cms de grosor, también realizadas en hormigón armado. La estructura y las vigas del edificio fueron hechas de tal manera que todo el complejo tiene un sistema de canales tubulares verticales y horizontales (a cada columna o viga le corresponde un vacío o canal adosada a la misma), de manera que todas las cañerías, cables eléctricos, etc. se instalaron allí. Desde los pasillos hay entradas a estos vacíos con puertas de una altura normal por lo que todas las obras de reparaciones pueden hacerse sin tener que entrar en las habitaciones, incluso las que afectan los sifones de los lavabos, que también están en dichos vacíos. El sanatorio está adecuado a los distintos tipos de pacientes, teniendo presente elementos como la visión de la vegetación exterior o el uso de una lámpara de luz indirecta en los pacientes postrados (Ver Figuras 14 y 15).

En el diseño de los interiores se cuidaron detalles como la introducción de superficies curvas en el encuentro de paredes y suelos para facilitar la limpieza, así como la elaboración de una escupidera especial para los enfermos, que finalmente no fue utilizada.

Este sanatorio fue concebido como un proyecto integral, lo que le llevó a la definición de un mobiliario específico. Producto de ello es la silla número 41 (Ver Figura 16), más tarde conocida como silla Paimio que, por su ergonómico diseño, favorecía la respiración del enfermo, dando evidencia que fue concebida bajo los ideales higienistas considerados en toda la obra.

Está inspirada en la silla tubular de acero "Wassily" (Ver Figura 17), de Marcel Breuer, pero sustituye el acero por la madera, ya que este material presentaba características más convenientes para un sanatorio; es de menor conducción térmica que el acero, no genera reflejos y absorbe el ruido, en lugar de contribuir a su propagación (Ver Figura 18).

Aalto tenía el mismo objetivo que buscaba la Bauhaus; el conocimiento de los materiales, de las posibilidades plásticas, de su aplicación tectónica. El conocimiento de los materiales permitió trabajar desde la dimensión funcional, dando respuesta a los requerimientos psicofísicos. Llevaba un tiempo investigando las posibilidades de curvatura de la madera laminada, a partir de ello, Aalto buscó nuevas formas de manipular la madera a partir del estudio de la construcción de los esquís, ampliando las posibilidades de su uso y creando una silla admirada tanto por su comodidad y fineza como por su presencia escultórica. Así, el material es curvado sin necesidad de cortes y uniones, similar al trabajo de Breuer en su silla. 


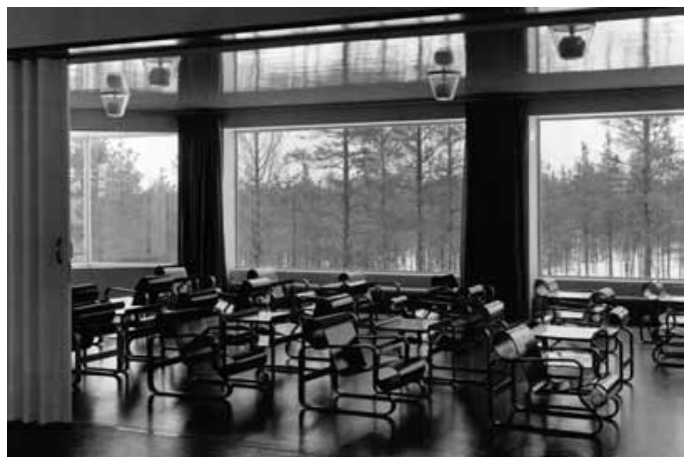

Figura 12.

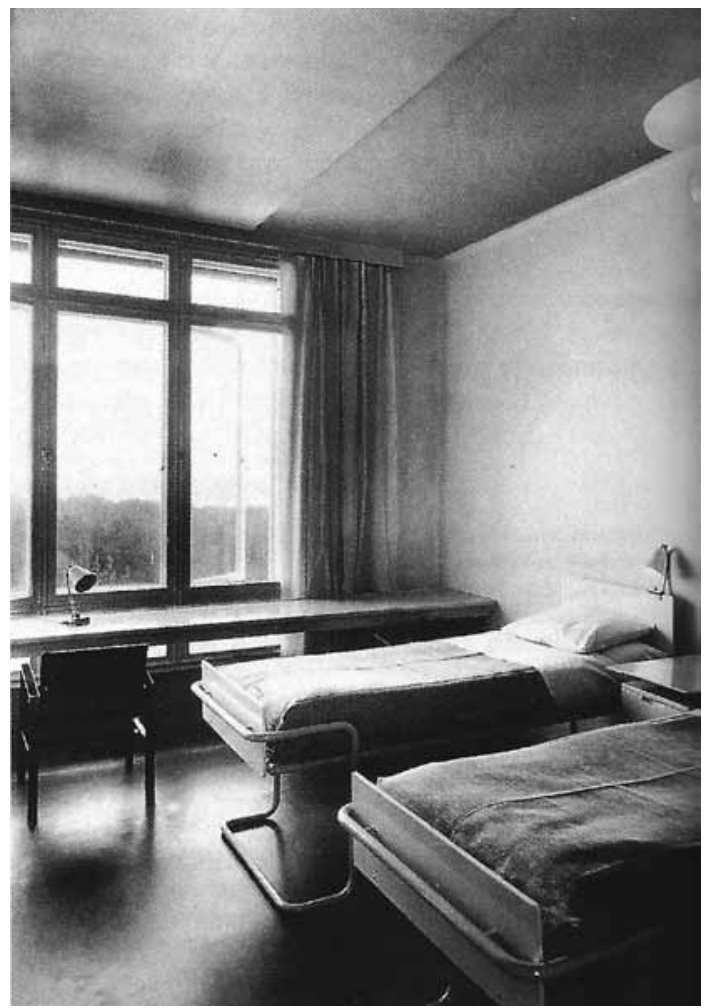

Figura 14.

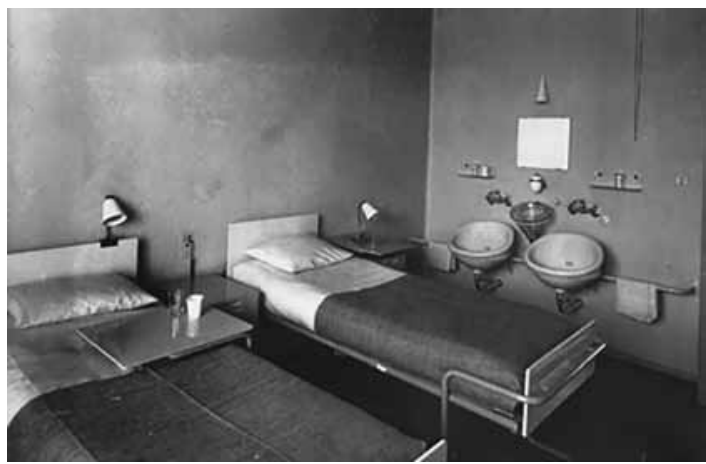

Figura 13.
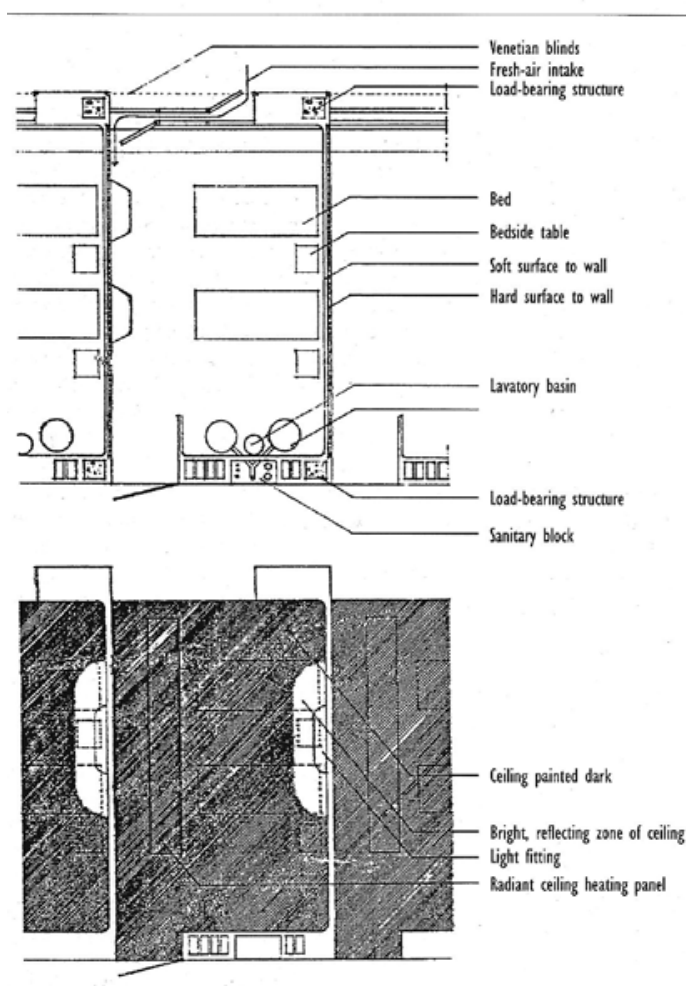

Figura 15. 


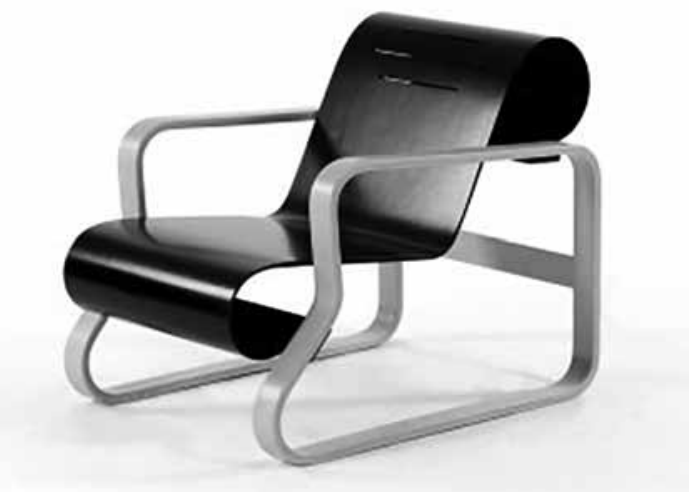

Figura 16.

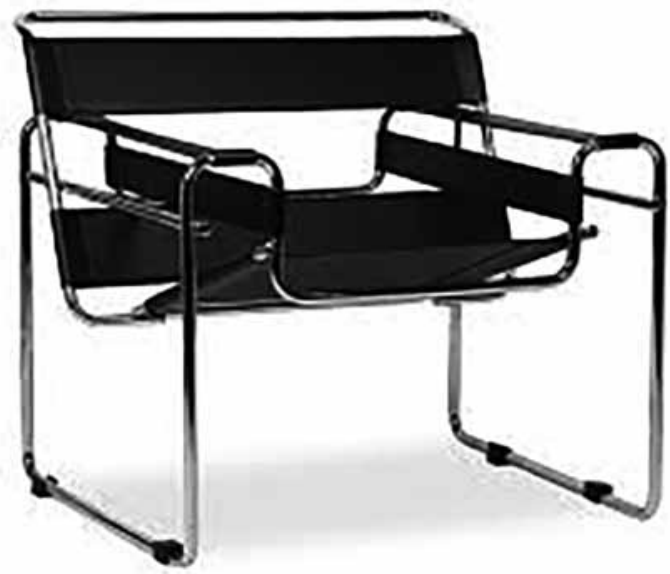

Figura 17.

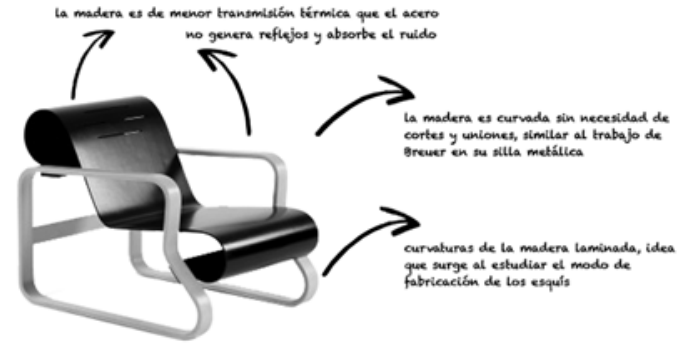

Figura 18.
Figura 12. Sala de recuperación del Sanatorio. Fuente: https:// es.wikiarquitectura.com/edificio/ sanatorio-antituberculoso-paimio/. Figura 13. Camas y lavabos en dormitorios del Sanatorio. Fuente: https://es.wikiarquitectura.com/ edificio/sanatorio-antituberculosopaimio/. Figuras 14 y 15. El mobiliario para el Sanatorio de Paimio se diseño con sentido práctico, funcional y de apoyo psicológico. El ventanal, en su parte inferior, dispone de un cristal a través del cual el enfermo tenía una vista de los jardines circundantes. Fuente: Schildt, Góren (1966). Alvar Aalto. A Life's Work-Architecture, Design and Art. Editorial Gustavo Gili. Figura 16. Silla Paimio de Aalto. Figura 17. Silla Wassily de Breuer. Fuente (Fig. 16 y 17): Jové Sandoval, J. (2003). Alvar Aalto; proyectando con la naturaleza. Valladolid; Universidad de Valladolid. Figura 18. Atributos de la Silla de Paimio. Fuente: Reproducción del autor. 


\section{Materialidad y morfología}

La idea constructiva de la silla Paimio, cuyo diseño se caracterizó por una modernidad elegante de tradición refinada, probablemente el más elegante de Aalto en esos años, buscaba manifestar el creciente bienestar a partir de la belleza cotidiana y el ensamble de lo mecánico con lo orgánico y de las formas abstractas del funcionalismo con los detalles vernáculos producidos por las tradiciones locales y el clima. Su condición morfológica consiste en una estructura laminar, realizada con delgadas tiras de madera contrachapada, sobre el que se coloca una lámina de madera curvada de abedul autóctono, tanto por sus propiedades y formas orgánicas como por su calidez y por compenetración con la naturaleza, generando el asiento y el respaldo con un solo gesto, pintado y pegado, de manera que su curvatura se ajusta a la forma del cuerpo humano, respondiendo a una búsqueda por facilitar la respiración del enfermo, y le dotan de la rigidez necesaria para su uso.

La silla mide $60 \mathrm{cms}$. de frente x $80 \mathrm{cms}$. de fondo x $64 \mathrm{cms}$. de alto y siendo para el descanso y sanación, resulta ser cómodo y elegante, sin embargo no es posible evitar el considerar que, por encima de los criterios funcionales y orgánicos, existe un fuerte sentido formal o plástico que le asigna un marcado valor simbólico. No obstante el uso de la silla queda sujeta, obviamente, a su función de manera que el utilizar la silla según lo señala Eco (2013, p. 326)

Es el ejercicio de una función por medio de un producto manufacturado que la promueve y la consiente: y decir que el producto manufacturado "promueve" la función, ya quiere decir que realiza una función comunicativa, que comunica la función que debe ser ejercida...

El material muestra la estructura y se transforma en el único componente estético con la simplicidad como regla, evocando una aplastante armonía sujeta a una geometría racional de líneas claras y sobrias que dejan al desnudo una precisión de las piezas y una claridad del montaje, lo que deriva en una cierta virtualidad de la forma en tanto su transparencia y levedad (Ver Figura 19). Con la continuidad como característica morfológica clave, que no se debe tan solo al empleo de un único material, sino a su elasticidad intrínseca y a su forma derivada. Los experimentos con el material lograron, por tanto, ahondar en las posibilidades de deformación y de utilización del tablero contrachapado como material adecuado para el diseño de mobiliario.

Consecuencia de ello la silla Paimio tiene gran similitud con la silla híbrida. Esta consiste en una cinta cortada longitudinalmente en dos finas tiras de madera, que se modelan con formas libres y serpenteantes, cuya relación con el asiento de la silla es evidente (Ver Figura 20). En esta silla, de gran fuerza plástica, Aalto manipula el asiento de madera, semejante al utilizado en otras ocasiones, pero realizando los reposabrazos con la misma pieza que forma el asiento. Para ello realiza dos cortes de la chapa de madera, de esta manera los laterales pueden curvarse en una dirección distinta al resto de la pieza. El ensayo participa del mismo mecanismo, realmente se trata de una única cinta, cortada longitudinalmente, justo hasta sus extremos que permanecen unidos. Así cada uno de sus partes, son deformadas, según curvaturas diferentes. 


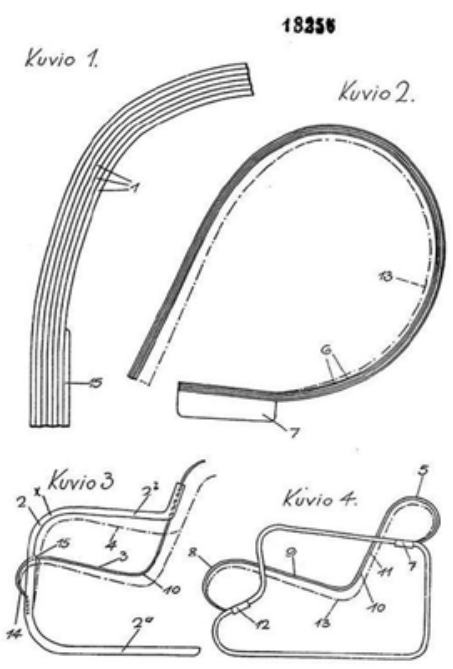

Figura 19.

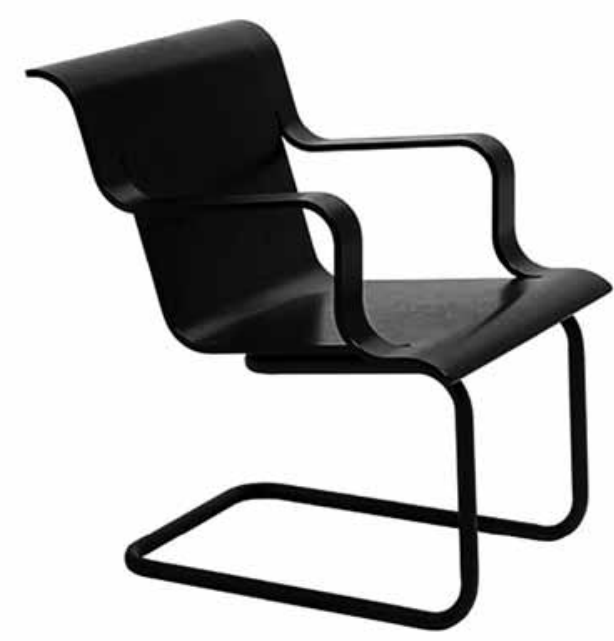

Figura 20.

Figura 19. Silla de Paimio, detalle constructivo. Fuente: Jové S., José María (2003). Alvar Aalto, proyectando con la naturaleza. Valladolid. Universidad de Valladolid. Figura 20. Silla Hibrida. Fuente: Jové S., José María (2003). Alvar Aalto, proyectando con la naturaleza. Valladolid. Universidad de Valladolid.

El sillón y la mesa auxiliar Paimío, en estas dos piezas se aplicó las propiedades del contrachapado que se había estado ensayando experimentalmente; flexibilidad y facilidad del material para ser moldeado. Con ellos se pudo formar las estrechas cintas para construir el bastidor de la silla, formando una pieza continua encolada. Sobre este bastidor se colocó el asiento de contrachapado curvado, que se utilizó en las sillas híbridas, moldeado de acuerdo a las dimensiones recomendables para su utilización, y según los requisitos psicofísicos.

\section{Conclusiones Provisorias}

En ese enfoque orgánico es crucial la manera en que los elementos individuales, como objetos o muebles, se conectan visual y funcionalmente con el contexto de su emplazamiento interior y el conjunto arquitectónico. Es así como el objeto orgánico -silla Paimio-,a vista del observador, y de acuerdo a su característica morfológica, es visualmente estimulante dando cabida a un conjunto de nociones presentes en su organización formal, enfatizando su coherencia integral y hace evidente, en ella, la síntesis formal obtenida por la asociación de todas las categorías funcionales -soporte, asiento, respaldo, apoyabrazos- a través de 
una superficie espacial soportada en un bastidor de forma casi cúbica, realizada con delgadas tiras de madera contrachapada. Se establece una clara categorización de los aspectos conformativos que constituyen la unidad, poniendo en evidencia las distintas categorías funcionales de lo cual se forma la silla.

El rasgo morfológico, del objeto, sustentado en la superficie laminar, espacial, simple, de simple curvatura, reconoce la posibilidad de sustentar un significado compartido, ya sea como entidad aislada, determinado por los aspectos conformativos y sus manifestaciones superficiales, y otro contextual -como objeto arquitectónico- en el que se relaciona con su entorno espacial, siendo capaz de comunicar a través de una mínima expresión de elementos, configurando un estado de virtualidad como modo de concreción.

Umberto Eco (2013, p. 324) afirma que "el objeto arquitectónico nos señala que por lo general disfrutamos de la arquitectura como acto de comunicación sin excluir su funcionalidad".

En contraposición con ello, en esta relación objeto espacialidad observada, se advierte la condición morfológica en una envolvente virtual, perceptualmente más pregnante que el conjunto de entidades materiales que la configuran. El perfil del objeto se desdobla en el espacio arquitectónico incrementando su intensidad perceptual, aumentando en consecuencia su prestancia y presencia; el objeto objeta el espacio y lo satura.

En esta condición comunicante, en su relación con la espacialidad que le acoge, podríamos afirmar que establece un modo de concreción en la relación polar virtualidad - saturación en donde la virtualización, según Peña (2012, p. 121), "se podría entender entonces como una mutación de identidad, un desplazamiento del centro de gravedad ontológico del objeto considerado, pero nunca como una reificación de la ilusión o lo falso".

\section{Notas}

1. La biomimética, se refiere a un campo de conocimiento que utiliza la naturaleza como fuente de inspiración o fuente de imitación para resolver ciertos problemas humanos que la naturaleza ya ha resuelto miles de años antes. En el diseño de productos, es una poderosa herramienta y fuente de inspiración sin fin, tratando de imitar las formas, texturas o funciones de los organismos vivos para alcanzar soluciones de diseño óptimas. Organic Design, Products Inspirated by Nature (2016). Send Point Publishing Co., Ltd.

2. La teoría hilemorfica planteada por Aristóteles, afirma que todas las substancia son un compuesto de dos coelementos distintos pero inseparables el uno del otro: la materia (hylé) y la forma (morphé).

3. Eliot Fette Noyes (1910-1977), arquitecto y diseñador industrial estadounidense formado en Harvard, trabajó en proyectos para IBM, especialmente en la máquina de escribir IBM Selectric y el Centro de Investigación Aeroespacial de IBM en Los Ángeles, California. Fue pionero en el desarrollo de programas integrales de diseño corporativo que integraron estrategia de diseño y estrategia comercial.

4. Søren Christian Hansen, fabricante danés de muebles. Durante la posguerra formó una moderna producción de muebles en la empresa familiar en colaboración con arquitectos de muebles, como Arne Jacobson. Hansen mismo diseñó algunos muebles. Las obras de 
estos arquitectos ayudaron a establecer el diseño danés como concepto en el exterior y a poner a Fritz Hansen S.A. en el mapa.

5. Fundado en 1874, Louis Poulsen es un fabricante de iluminación danés nacido de la tradición del diseño escandinavo, donde la forma sigue la función. La función y el diseño de sus productos están diseñados para reflejar y apoyar el ritmo de la luz natural. Cada detalle en el diseño tiene un propósito. Cada diseño comienza y termina con luz. https:// www.louispoulsen.com/usa/about-us/about-louis-poulsen/

6. Zonnestraal, es el nombre de un complejo hospitalario situado en la ciudad holandesa de Hilversum. Fue diseñado y construido por los arquitectos holandeses, Jan Duiker y Bernard Bijvoet en forma progresiva entre los años 1919 y 1940.

\section{Referencias}

Araya,V.; Alfaro, M. \& Andonegui, M. (2007). Constructivismo; orígenes y perspectivas. Laurus, 13(24), 76-92.

ARQHYS. (2012). Sanatorio Paimio de Alvar Aalto. Revista ARQHYS.com. Obtenido de http://www.arqhys.com/sanatorio-paimio-de-alvar-aalto.html.

Eco, U. (2013). La estructura ausente. Buenos Aires: Edit. De bolsillo.

Jové Sandoval, J. (2003). Alvar Aalto; proyectando con la naturaleza. Valladolid; Universidad de Valladolid.

Lévy, P. (1999). ¿Qué es lo virtial? Barcelona: Paidos.

Lago García, C. (2007). Antropología filosófica; el ser humano como problema filosófico. Centro de Integración Tecnológica para el Aprendizaje (CITA). Escuela de Artes Plásticas de Puerto Rico. Obtenido de: http://cita.eap.edu/moodle/pluginfile.php/1831/mod_resource /content/1/Filosofia/Antropologia_Filosofica.pdf

Peña, W. (2012). La virtualidad como lugar para la reflexión. Reflexiones teológicas 8 (119 - 130). Bogotá, Colombia.

Pokropek, J. \& Amoroso, J. (S/F). Revisando nuestra morfología arquitectónica en función del mensaje estético. Obtenido de: http://wiki.fadu.uba.ar/images/d/d8/Revisando_"nuestra"_ morfolog\%C3\%ADa_arquitectónica_en_función_del_mensaje_estético._Desde_ la_“Teor\%C3\%ADa_del_habitar"_a_"Las_expresiones_de_la_Arquitectura”.pdf

Pokropek, J. \& Cravino, A. (2018). Algunas precisiones sobre la borrosa noción de "Materia" para el diseño interior. Cuaderno 70. Centro de Estudios en Diseño y Comunicación. pp. $15-27$.

Rios, S. (2014). Convivencias entre el arte y el diseño; el resurgir del diseño en la década de los cincuenta y su expansión hacia la cultura de lo cotidiano. Tsantsa. Revista de Investigaciones Artísticas Núm. 1- (Año, 2014) ISSN: 1390-8448).

Ruiz Trujillo, P. (2015). Aristóteles, De la potencia al acto. Bonalletra Alcompas, S. L.

Sanatorio Antituberculoso Paimio. Obtenido de: https://es.wikiarquitectura.com/edificio/ sanatorio-antituberculoso-paimio/

Schildt, G. (1996). Alvar Aalto; obra completa: arquitectura, arte y diseño. Barcelona: Gustavo Gili, S. A.

Organic Design, Products Inspirated by Nature (2016). Send Point Publishing Co., Ltd. 
Designaholic (2017). La importancia de la silla serie 7. Obtenido de: http://designaholic. $\mathrm{mx} / 2017 / 09 /$ iho-espacios-presenta-la-importancia-la-silla-serie-7-la-historia-deldiseno.html

Olguin, G.; Castellano, L.; Abraham, M.; Bourdichon, M.; De La Fuente, F.; Giurdanella, S.; Hernández, T.; Scocco, M. (2009). Leer la Forma; estudio sobre la morfología aplicada al diseño . 1ra. Edición. Buenos Aires: Redargenta Ediciones.

\begin{abstract}
From the organic architecture, understood as a philosophy that promotes the harmony between the human habitat and the natural environment, marks a substantial break with the traditional formal compositions, and from where the properties were taken of new materials and new productive techniques. This movement arises in opposition rationalism or functionalism, manifesting itself in the first decades of the 20th century and with this the design of objects is compromised and particularly the furniture design of interiors, based on organic structures, and a form of design that opens borders between the material and the sentimental, whose source of experiences is nature. The great diversity of solutions -present in our natural environment- to structural problems, in terms of energy and functionality, they have already been tested for millions of years, and in it we can find all kinds of patterns and combinations of shapes, colors and textures that are the foundation of organic design as a source inexhaustible inspiration for designers. It is constituted as a powerful tool and endless source of inspiration, united by the principles of life itself, trying to imitate the forms, textures or functions of living organisms to reach solutions of optimal design.

In the present work a brief tour through the beginnings of organic design is shown, particularly furniture design, going through some representative classics of the Nordic and American design, from the form-matter relationship.

Then one of the main exponents of organic design is recognized: Alvar Aalto, Finnish architect and designer, through two of his most iconic creations; the project Sanatorio de Paimio and the chair 41. The central idea of the Sanatorium was a building that favored the healing and rehabilitation of tuberculosis patients. East The project was conceived integrally, which led to the definition of a specific furniture. Product of it is chair number 41, later known as chair Paimio de which realizes its morphological and material characteristics. Finally I know establishes a relationship where the object connects visually and functionally with the context of its interior location of the architectural ensemble.
\end{abstract}

Keywords: Organicism - organic design - Paimio Sanatorium - chair 41 - Aalvar Alto.

Resumo: Da arquitetura orgânica, entendida como uma filosofia que promove a harmonia entre o habitat humano e o ambiente natural, marca uma ruptura substancial com as composições formais tradicionais, e de onde as propriedades foram tomadas de novos materiais e novas técnicas produtivas. Este movimento surge em oposição racionalismo ou funcionalismo, manifestando-se nas primeiras décadas do século XX e com isso, o design de objetos é comprometido e, particularmente, o design de móveis de interiores, 
baseados em estruturas orgânicas, e uma forma de design que abre fronteiras entre o material e o sentimental, cuja fonte de experiências é a natureza. A grande diversidade de soluções -presentes em nosso ambiente natural- para problemas estruturais, em termos de energia e funcionalidade, eles já foram testados por milhões de anos, e nele podemos encontrar todos os tipos de padrões e combinações de formas, cores e texturas que são a base do design orgânico como fonte inspiração inesgotável para designers. É constituído como uma ferramenta poderosa fonte inesgotável de inspiração, unida pelos princípios da própria vida, tentando imitar as formas, texturas ou funções dos organismos vivos para alcançar soluções de design ideal.

No presente trabalho, um breve passeio pelos primórdios do design orgânico é mostrado, particularmente design de móveis, passando por alguns clássicos representativos do Design nórdico e americano, a partir da relação forma-matéria. Então um dos principais expoentes do design orgânico é reconhecido: Alvar Aalto, Arquiteto e designer finlandês, através de duas de suas criações mais icônicas; o projeto Sanatório de Paimio e a cadeira 41. A ideia central do Sanatório foi um edifício que favoreceu a cura e reabilitação de pacientes com tuberculose. Este aqui $O$ projeto foi concebido integralmente, o que levou à definição de um mobiliário específico. Produto dele é a cadeira número 41, mais tarde conhecida como cadeira Paimio de que percebe suas características morfológicas e materiais. Finalmente, estabelece um relacionamento onde o objeto se conecta visual e funcionalmente com o contexto da sua localização interior do conjunto arquitetônico.

Palavras-chave: Organicism - design orgânico - Sanatorio Paimio - cadeira 41 - Aalvar.

[Las traducciones de los abstracts fueron supervisadas por el autor de cada artículo] 